\title{
Impacto dos Índices Aterogênicos em Estenose do Enxerto de Veia Safena
}

\author{
Impact of Atherogenic Indexes in Saphenous Vein Graft Stenosis
}

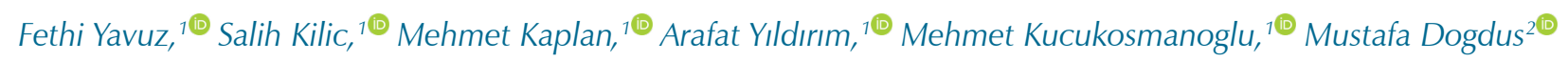
Health Sciences University, Adana Research and Training Hospital, Department of Cardiology, ${ }^{1}$ Adana - Turquia

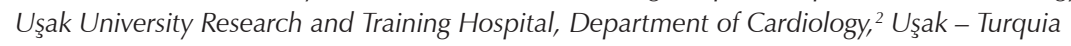

\section{Resumo}

Fundamento: Os enxertos de veias safenas (EVS) são frequentemente usados em pacientes submetidos a cirurgia de revascularização do miocárdio (CRM).

Objetivos: Avaliar as relações entre índices aterogênicos e estenose de EVS. Métodos: No total, 534 pacientes (27,7\% mulheres, com idade média de $65 \pm 8,4$ anos) submetidos a CRM e angiografia coronariana eletiva foram inclú́dos no estudo. Pacientes com pelo menos uma estenose EVS $\geq 50 \%$ foram alocados ao grupo estenose EVS $(+)(n=259)$ e pacientes sem estenose foram classificados como EVS (-) $(n=275)$. $O$ índice aterogênico plasmático (IAP) e o coeficiente aterogênico (CA) foram calculados a partir dos parâmetros lipídicos de rotina dos pacientes. A significância foi estabelecida no nível p<0,05.

Resultados: O número de pacientes com histórico de hipertensão (HT), diabetes mellitus (DM), acidente vascular cerebral e insuficiência cardíaca (IC) se mostrou significativamente maior no grupo EVS (+) do que no grupo EVS (-). O colesterol total, triglicerídeos e colesterol LDL mostraram-se significativamente mais altos e o colesterol HDL mostrou-se menor no grupo EVS $(+)$ do que no grupo EVS $(-)$. IAP $(p<0,001)$ e CA $(p<0,001)$ apresentaram-se significativamente mais altos no grupo EVS (+) do que no grupo EVS (-). A análise ROC mostra que tanto o IAP quanto o CA mostraram-se melhores que o colesterol HDL, colesterol LDL e colesterol não HDL na predição de estenose de EVS. Na análise multivariada, histórico de DM, HT, acidente vascular cerebral, IC, número de enxertos de safena, colesterol HDL, colesterol LDL, colesterol não HDL, IAP e CA foram fatores de risco independentes para estenose de EVS.

Conclusão: O IAP e o CA foram preditores independentes de estenose de EVS. Além disso, tanto o IAP quanto o CA têm melhor desempenho na predição de estenose de EVS do que o colesterol LDL, colesterol HDL e colesterol não HDL. (Arq Bras Cardiol. 2020; 115(3):538-544)

Palavras-chave: Veia Safena/transplante; Veia Safena/estenose; Aterosclerose; Placa Aterosclerótica; LDL Colesterol; HDL Colesterol; Hipertensão; Diabetes Mellitus; Acidente Vascular Cerebral.

\begin{abstract}
Background: Saphenous vein grafts (SVG) are frequently used in patients that have undergone coronary artery bypass graft (CABG) surgery. Objectives: To evaluate the relationship between atherogenic indexes and SVG stenosis.

Methods: Altogether, 534 patients (27.7\% women, mean age $65 \pm 8.4$ years) that underwent CABG and elective coronary angiography were included in the study. Patients with at least one SVG stenosis $\geq 50 \%$ were allocated to the stenosis group SVG $(+)(n=259)$ and patients without stenosis were categorized as SVG (-) $(n=275)$. Atherogenic index of plasma (AIP) and atherogenic coefficient (AC) were calculated from the patients' routine lipid parameters. The level of significance was $p<0.05$.

Results: The number of patients with a history of hypertension (HT), diabetes mellitus (DM), stroke, and heart failure was significantly higher in the SVG (+) group than in the SVG (-) group. Total cholesterol, triglycerides, LDL-C were significantly higher and HDL-C was lower in the SVG (+) group than in the SVG (-) group. AIP $(p<0.001)$ and AC $(p<0.001)$ were significantly higher in the SVG $(+)$ group than in the SVG (-) group. The receiver operating characteristic (ROC) analysis show that both AIP and AC were better than HDL-C, LDL-C and non-HDL-C at predicting SVG stenosis. In the multivariate analysis, history of DM, HT, stroke, heart failure (HF), number of saphenous grafts, HDL-C, LDL-C, non-HDL-C, AIP and AC were found to be independent risk factors for SVG stenosis.
\end{abstract}

Conclusion: AIP and AC were independent predictors of SVG stenosis. Moreover, both AIP and AC have better performance in predicting SVG stenosis than LDL-C, HDL-C and non-HDL-C. (Arq Bras Cardiol. 2020; 115(3):538-544)

Keywords: Saphenous Vein/transplantation; Saphenous Vein/Stenosis; Plaque, Atherosclerotic; LDL, Cholesterol; HDL, Cholesterol; Hypertension; Diabetes Mellitus; Stroke

Full texts in English - http://www.arquivosonline.com.br

Correspondência: Fethi Yavuz •

Health Sciences University, Adana Research and Training Hospital, Department of Cardiology, Adana, Turkey - Department of Cardiology - Kışla Mahallesi, Dr. Mithat Özsan Bulvarı, 4522. Sokak No:1, Yüreğir/Adana Adana 01220 - Turquia

E-mail: fethiyavuz782@gmail.com

Artigo recebido em 03/10/2019, revisado em 03/12/2019, aceito em 27/12/2019

DOI: https://doi.org/10.36660/abc.20190683 


\section{Introdução}

Embora a cirurgia de revascularização do miocárdio (CRM) tenda a usar enxertos arteriais, os enxertos de veias safenas (EVS) ainda são frequentemente utilizados, principalmente em situações de emergência e em lesões de múltiplos vasos. ${ }^{1} \mathrm{O}$ EVS tende a degenerar com o tempo. A deficiência de EVS limita o sucesso a longo prazo da cirurgia de revascularização miocárdica. As taxas de patência no 1으, 5 e no 10ํano após a CRM foram 93\%, 74\% e $41 \%$, respectivamente. ${ }^{2}$

As principais causas de deficiência de EVS são trombose no período inicial ( $<1$ mês), hiperplasia neointimal no período subagudo (1-12 meses) e aterosclerose no período tardio. ${ }^{3-5}$

Sabe-se que os níveis lipídicos séricos estão fortemente associados à aterosclerose. ${ }^{6}$ Níveis elevados de colesterol de lipoproteína de alta densidade (colesterol HDL) proporcionam um efeito cardioprotetor, enquanto níveis elevados de colesterol de lipoproteína de baixa densidade (colesterol LDL) são aterogênicos. Além disso, muitos estudos demonstraram que altos níveis de triglicerídeos (TG) estão associados à doença arterial coronariana (DAC). ${ }^{7-9}$ No entanto, a relação entre aumento dos parâmetros lipídicos e aumento do risco de doença arterial coronariana (DAC) permanece controversa. Estudos anteriores que mostram a associação entre colesterol e aterosclerose foram realizados usando os níveis de colesterol total (CT) e LDL-C. Quando do surgimento do papel do colesterol HDL como agente cardioprotetor, o uso da razão colesterol LDL/colesterol HDL foi recomendado para determinar o risco de DAC. Como os efeitos dos níveis séricos elevados de TG na aterosclerose estão claramente definidos, essa razão tem valor limitado, pois não inclui os níveis de TG. ${ }^{10}$

É importante ressaltar que diversas razões de lipoproteínas ou índices aterogênicos podem ser usados para otimizar a capacidade preditiva do perfil lipídico. Estudos demonstraram que os índices lipídicos calculados a partir dos parâmetros do perfil lipídico apresentam melhor valor preditivo em doenças cardiovasculares. ${ }^{11} \mathrm{O}$ colesterol não HDL é recomendado como um preditor mais provável de DAC do que o colesterol LDL, porque representa o teor de colesterol encontrado em todas as lipoproteínas aterogênicas. ${ }^{12} \mathrm{O}$ índice aterogênico plasmático (IAP) é calculado por meio de dois parâmetros importantes: TG sérico e colesterol HDL sérico. O uso simultâneo de triglicerídeos e colesterol HDL para essa razão reflete múltiplas interações entre o metabolismo de diferentes lipoproteínas e pode ser útil para predizer a aterogenicidade plasmática. ${ }^{13}$ Outro índice é o coeficiente aterogênico (CA) calculado como a razão entre colesterol não HDL e HDL. ${ }^{14}$ Até onde sabemos, a associação de índices aterogênicos plasmáticos com doença de EVS não foi estudada. Neste estudo, investigamos a relação entre os parâmetros lipídicos e os índices aterogênicos IAP e CA na estenose de EVS.

\section{Materiais e Métodos}

O estudo incluiu 534 pacientes $(27,7 \%$ mulheres, $n=148)$ submetidos a angiografia coronariana eletiva por mais de um ano (média de 5,3 anos) após a cirurgia de revascularização miocárdica, que envolveu pelo menos um EVS para bypass. Os pacientes foram divididos em dois grupos de acordo com a extensão da patência do EVS. Estenose de $50 \%$ ou mais, dentro do EVS, foi categorizada como estenose de EVS (+).
O comitê de ética local aprovou o protocolo deste estudo, conduzido de acordo com os princípios estabelecidos na Declaração de Helsinque (22/05/2019).

As características clínicas e demográficas basais da população do estudo foram registradas no formulário preparado para cada paciente. Todos os parâmetros laboratoriais de rotina antes da angiografia coronariana, laudo ecocardiográfico, peso, altura e índice de massa corporal (IMC) foram registrados no sistema digital do hospital. Calculou-se o IMC como peso/altura $\left(\mathrm{m}^{2}\right)$. A hipertensão foi definida como medidas sistêmicas repetidas da pressão arterial que excederam 140/90 mmHg ou se o paciente estivesse tomando medicação anti-hipertensiva. Diagnosticouse diabetes mellitus (DM) de acordo com um dos seguintes critérios: (1) glicemia em jejum $\geq 126$ mg/dL, (2) glicemia > 200 $\mathrm{mg} / \mathrm{dL}$ a qualquer momento, (3) histórico de DM ou pacientes sob medicação antidiabética. Definiu-se hipercolesterolemia como nível basal total de colesterol $>200 \mathrm{mg} / \mathrm{dL}$ ou tratamento atual com estatinas e/ou agentes hipolipemiantes. Fumantes atuais foram os que relataram tabagismo regular nos 6 meses anteriores. Definiu-se histórico familiar de doença arterial coronariana como a presença de doença arterial coronariana em parente de primeiro grau masculino com idade $<55$ anos ou em parente de primeiro grau feminino com idade $<65$ anos.

\section{Cálculo de Îndices Aterogênicos}

O colesterol não HDL foi calculado como a diferença entre colesterol total e colesterol de alta densidade (colesterol total sérico - HDL-C sérico). ${ }^{15}$

O IAP foi calculado como o logaritmo da razão sérica de triglicerídeos/colesterol HDL sérico. ${ }^{13,16}$

O coeficiente aterogênico (CA) foi calculado como a razão entre colesterol não HDL e HDL, da seguinte forma: (colesterol sérico total — HDL-C sérico)/colesterol HDL). ${ }^{14}$

\section{Avaliação Angiográfica}

A angiografia coronariana foi realizada rotineiramente pela técnica de Judkins, com 6 ou 7 cateteres cardíacos franceses direito e esquerdo. Os angiogramas foram gravados em mídia digital DICOM a 25 quadrados/ms e foram revisados por dois angiógrafos experientes que desconheciam o estado clínico dos pacientes. Os enxertos de veias safenas foram vistos a partir de pelo menos dois ângulos após injeção seletiva de material de contraste. Definiu-se doença de enxerto venoso como estenose de $50 \%$ do diâmetro do vaso em qualquer EVS.

\section{Análise Estatística}

Variáveis contínuas com distribuição normal foram descritas como média \pm desvio padrão (DP) e variáveis sem distribuição normal foram descritas como mediana e intervalo interquartil. As variáveis categóricas são apresentadas como o número de pacientes e porcentagens. As variáveis foram investigadas pelo método analítico de Kolmogorov-Smirnov para determinar se apresentam distribuição normal ou não. Realizou-se teste $t$ de Student não pareado para variáveis com distribuição normal e o teste $U$ de Mann-Whitney para aquelas sem distribuição normal. Para as variáveis categóricas, utilizou-se o teste do qui-quadrado ou o exato de Fisher. O tamanho da amostra da população do 
estudo não foi calculado e todos os pacientes consecutivos em nossa clínica foram incluídos no estudo.

Foram utilizadas curvas receiver operating characteristic (ROC) para demonstrar a sensibilidade e especificidade dos índices e lipídios, e seus valores de corte para prever estenose de EVS. A área sob a curva (AUC) desses índices aterogênicos e lipídios foi calculada pelo método DeLong. ${ }^{17}$ Para calcular as hazard ratios (HR) e seus intervalos de confiança de 95\% (IC 95\%) para estenose de EVS, realizou-se análise de regressão logística univariada e multivariável. Valores de $p<0,05$ foram considerados estatisticamente significativos. Os dados foram analisados utilizando o software estatístico SPSS, versão 20.0 (SPSS Inc., Chicago, IL, EUA) e o software estatístico MedCalc 15 (Ostend, Bélgica).

\section{Resultados}

No total, 534 pacientes (idade média de $65 \pm 8,4$ anos) foram incluídos no estudo. Desse número, 259 (48,5\%) foram diagnosticados com estenose de EVS (EVS (+)) e 275 $(51,5 \%)$ foram diagnosticados com patência de EVS (EVS $(-))$. As características demográficas e clínicas basais dos grupos estudados estão resumidas na Tabela 1. O número de pacientes com histórico de HT, DM, acidente vascular cerebral e insuficiência cardíaca se mostrou significativamente maior no grupo estenose EVS (+) do que no grupo estenose EVS (-). A pressão arterial sistólica média e o número de enxertos foram maiores no grupo estenose EVS (+) do que no grupo estenose EVS (-). A fração de ejeção ventricular esquerda foi menor no grupo estenose EVS (+) do que no grupo estenose SVG (-). Os parâmetros laboratoriais e os índices lipídicos dos grupos de pacientes são apresentados na Tabela 2. Os parâmetros lipídicos, i.e., colesterol total, triglicerídeos e colesterol LDL apresentaram-se significativamente maiores, e o colesterol HDL apresentou-se menor no grupo EVS (+) do que no grupo EVS (-). Os índices lipídicos, IAP, CA e colesterol não HDL mostraram-se significativamente mais altos no grupo estenose EVS (+) do que no grupo estenose EVS (-) ( $p<0,001$, para todos).

Para a predição de estenose de EVS, o valor de corte de $3,4<$ CA possui sensibilidade de $79,15 \%$ e especificidade de $66.18 \%$; o valor de corte de $0.56<$ IAP possui $83,01 \%$ de sensibilidade e $67,53 \%$ de especificidade; colesterol $\mathrm{HDL}<40 \mathrm{mg} / \mathrm{dL}$ possui $64,9 \%$ de sensibilidade e $84,0 \%$ de especificidade; $121 \mathrm{mg} / \mathrm{dL}<$ colesterol LDL possui $78,4 \%$ de sensibilidade e 58,9\% de especificidade; e $141<$ colesterol não HDL possui $70,66 \%$ de sensibilidade e $54,55 \%$ de especificidade nas análises da curva ROC (Figura). Comparações pareadas da análise ROC mostram que, embora não houvesse diferenças significativas entre o IAP e o CA, tanto o IAP quanto o CA mostraram-se superiores ao colesterol HDL, colesterol LDL e colesterol não HDL na predição de estenose de EVAS.

Para determinar os fatores de risco independentes para estenose de EVS, realizou-se análise logística univariada e multivariada (Tabela 3). Na análise multivariada, histórico de $\mathrm{DM}, \mathrm{HT}$, acidente vascular cerebral, IC, número de enxertos de safena, colesterol HDL, colesterol LDL e colesterol não HDL foram fatores de risco independentes para estenose de EVS. Além disso, os índices aterogênicos IAP e CA foram preditores independentes de estenose de EVS. Embora o nível
Tabela 1 - Comparação das características basais clínicas da população estudada

\begin{tabular}{|c|c|c|c|}
\hline Variável & $\begin{array}{l}\text { Estenose de } \\
\text { EVS (+) } \\
(\mathrm{n}=259)\end{array}$ & $\begin{array}{l}\text { Estenose de } \\
\text { EVS }(-) \\
(n=275)\end{array}$ & $p$ \\
\hline Idade, anos & $65,9 \pm 8,66$ & $64,6 \pm 8,22$ & 0,071 \\
\hline Índice de massa corporal, kg/m² & $28,1 \pm 3,75$ & $28,0 \pm 4,70$ & 0,709 \\
\hline Pressão arterial sistólica, mmHg & $126,5 \pm 17,55$ & $123,0 \pm 12,24$ & 0,007 \\
\hline Pressão arterial diastólica, mmHg & $75,2 \pm 9,89$ & $74,0 \pm 8,38$ & 0,141 \\
\hline Intervalo após a cirurgia, mês & $67,5 \pm 39,55$ & $60,6 \pm 29,53$ & 0,171 \\
\hline $\begin{array}{l}\text { Fração de ejeção ventricular } \\
\text { esquerda, \% }\end{array}$ & $52,1 \pm 9,48$ & $55,8 \pm 6,38$ & $<0,001$ \\
\hline Sexo feminino, \% (n) & $25,1(65)$ & $30,4(83)$ & 0,172 \\
\hline Hipertensão, \% (n) & $78,0(202)$ & $66,5(183)$ & 0,003 \\
\hline Diabetes mellitus, \% (n) & $62,5(162)$ & $46,5(128)$ & $<0,001$ \\
\hline Hiperlipidemia, \% (n) & $77,6(201)$ & $76,2(204)$ & 0,356 \\
\hline Doença vascular periférica, \% (n) & $12,7(33)$ & $9,5(26)$ & 0,226 \\
\hline Acidente vascular cerebral, \% (n) & $17,8(46)$ & $8,7(24)$ & 0,002 \\
\hline Doença renal crônica, \% (n) & $3,9(10)$ & $2,9(8)$ & 0,542 \\
\hline Insuficiência cardíaca, \% (n) & $38,6(100)$ & $15,6(43)$ & $<0,001$ \\
\hline Fibrilação atrial, \% (n) & $6,2(16)$ & $6,6(18)$ & 0,827 \\
\hline Número de enxertos & $1,99 \pm 0,7$ & $1,7 \pm 0,65$ & $<0,001$ \\
\hline Aspirina, \% (n) & $81,1(210)$ & $85,8(236)$ & 0,140 \\
\hline Inibidores de P2Y12, \% (n) & $32,4(84)$ & $29,5(81)$ & 0,457 \\
\hline Betabloqueadores, \% (n) & $87,6(227)$ & $85,1(234)$ & 0,391 \\
\hline IECA/BRA, \% (n) & $75,7(196)$ & $71,6(197)$ & 0,290 \\
\hline Estatina, \% (n) & $59,8(155)$ & $64,7(178)$ & 0,245 \\
\hline Diuréticos, \% (n) & $22,8(59)$ & $15,3(42)$ & 0,027 \\
\hline Trimetazidina, \% (n) & $24,3(63)$ & $27,3(75)$ & 0,437 \\
\hline Ranolazina, \% (n) & $17,8(46)$ & $18,9(52)$ & 0,732 \\
\hline Nitratos, \% (n) & $27,8(72)$ & $16,7(46)$ & 0,002 \\
\hline $\begin{array}{l}\text { Bloqueadores de canais de } \\
\text { cálcio, \% (n) }\end{array}$ & $18,9(49)$ & $19,6(54)$ & 0,834 \\
\hline
\end{tabular}

IECA: Inibidor da enzima conversora da angiotensina; BRA: bloqueador do receptor de angiotensina.

de triglicerídeos tenha sido significativo na análise univariada, ele não alcançou significância estatística na análise multivariada.

\section{Discussão}

No presente estudo, observamos que fatores de risco cardiovascular tradicionais, como HT, DM, acidente vascular cerebral e insuficiência cardíaca, foram preditores independentes de estenose de EVS. Além disso, descobrimos que o colesterol $\mathrm{HDL}$, colesterol LCL, colesterol não $\mathrm{HDL}$, número de EVS e os índices aterogênicos IAP e CA foram preditores independentes de estenose de EVS. Além disso, observamos que o IAP e o CA têm melhor desempenho na predição de estenose de EVS do que o colesterol LDL, colesterol HDL e colesterol não HDL. 
Tabela 2 - Comparação de parâmetros laboratoriais e índices lipídicos dos grupos de estudo

\begin{tabular}{|c|c|c|c|}
\hline Variável & $\begin{array}{l}\text { Estenose de } \\
\text { EVS }(+)(n=259)\end{array}$ & $\begin{array}{l}\text { Estenose de EVS } \\
\quad(-)(n=275)\end{array}$ & $p$ \\
\hline $\mathrm{Hba1c}, \%$ & $7,2 \pm 1,9$ & $7,0 \pm 2,0$ & 0,296 \\
\hline Glicemia de jejum, md/dL & $174 \pm 108$ & $164 \pm 94$ & 0,249 \\
\hline Hemoglobina, g/dL & $13,2 \pm 1,64$ & $13,1 \pm 1,84$ & 0,205 \\
\hline Hematócrito, \% & $39,1 \pm 4,82$ & $38,6 \pm 4,97$ & 0,259 \\
\hline $\begin{array}{l}\text { Contagem de glóbulos } \\
\text { brancos, } x 10^{3} / \mathrm{mL}\end{array}$ & $8,7 \pm 2,26$ & $8,5 \pm 2,1$ & 0,553 \\
\hline $\begin{array}{l}\text { Contagem de plaquetas, } \\
\times 10^{3} / \mathrm{mL}\end{array}$ & $244 \pm 68$ & $237 \pm 65$ & 0,206 \\
\hline $\begin{array}{l}\text { Contagem de neutrófilos, } \\
\times 10^{3} / \mathrm{mL}\end{array}$ & $5,5 \pm 1,78$ & $5,3 \pm 1,93$ & 0,307 \\
\hline $\begin{array}{l}\text { Contagem de linfócitos, } \\
\times 10^{3} / \mathrm{mL}\end{array}$ & $2,1 \pm 0,8$ & $2,1 \pm 0,7$ & 0,377 \\
\hline $\begin{array}{l}\text { Contagem de monócitos, } \\
\times 10^{3} / \mathrm{mL}\end{array}$ & $0,7 \pm 0,21$ & $0,7 \pm 0,26$ & 0,860 \\
\hline Volume médio plaquetário, fl & $8,8 \pm 0,96$ & $9,9 \pm 1,12$ & 0,123 \\
\hline Red cell distribution width, $\mathrm{fL}$ & $15,7 \pm 2,71$ & $14,7 \pm 2,14$ & 0,182 \\
\hline $\begin{array}{l}\text { Colesterol total, mg/dL, } \\
\text { mediano (percentil } 25^{\circ}-75^{\circ} \text { ) }\end{array}$ & 201 (174-232) & $185,0(165-222)$ & 0,001 \\
\hline Colesterol LDL, mg/dL & $137,0 \pm 34,7$ & $126,8 \pm 37,2$ & 0,001 \\
\hline $\begin{array}{l}\text { Colesterol HDL, mg/dL, } \\
\text { mediano (percentil } 25^{\circ}-75^{\circ} \text { ) }\end{array}$ & $37,0(34,0-43,0)$ & $48,0(42-54,5)$ & $<0,001$ \\
\hline $\begin{array}{l}\text { Triglicerídeos, mg/dL, } \\
\text { mediano (percentil } 25^{\circ}-75^{\circ} \text { ) }\end{array}$ & $203(156-273)$ & $143,0(111-189,5)$ & $<0,001$ \\
\hline $\begin{array}{l}\text { Ureia, mg/dL, } \\
\text { mediana (percentil } 25^{\circ}-75^{\circ} \text { ) }\end{array}$ & $37,0(29,0-47,0)$ & $37,0(29,0-42,0)$ & 0,621 \\
\hline $\begin{array}{l}\text { Creatinina, mg/dL, } \\
\text { mediana (percentil } 25^{\circ}-75^{\circ} \text { ) }\end{array}$ & $0,90(0,70 .-1.10)$ & $0,80(0,70-1,0)$ & 0,253 \\
\hline Albumina, mg/dL & $3,8 \pm 0,49$ & $3,9 \pm 0,38$ & 0,174 \\
\hline Alanina transaminase, $\mathrm{U} / \mathrm{L}$ & $19,1 \pm 2,93$ & $19,0 \pm 3,17$ & 0,899 \\
\hline $\begin{array}{l}\text { Aspartato } \\
\text { aminotransferase, U/L }\end{array}$ & $23,2 \pm 3,61$ & $24,2 \pm 3,94$ & 0,966 \\
\hline Ácido úrico sérico, mg/dL & $5,7 \pm 1,6$ & $5,4 \pm 1,7$ & 0,172 \\
\hline IAP & $0,73 \pm 0,20$ & $0,50 \pm 0,23$ & $<0,001$ \\
\hline $\mathrm{CA}$ & $4,44 \pm 1,30$ & $3,08 \pm 1,06$ & $<0,001$ \\
\hline Colesterol não HDL & $165,7 \pm 42,74$ & $144,6 \pm 44,44$ & $<0,001$ \\
\hline
\end{tabular}

CA: Coeficiente aterogênico; IAP: Índice aterogênico plasmático; $H D L$ : Lipoproteína de alta densidade, LDL: Lipoproteína de baixa densidade.

A estenose do enxerto de veia safena é uma questão importante no curto e no longo prazo dos pacientes submetidos a CRM, estando relacionada a grandes eventos cardiovasculares adversos. É importante identificar pacientes com alto risco de estenose de EVS para estratégias de prevenção e tratamento. Muitos fatores de risco relacionados à estenose de EVS, como fatores cirúrgicos, tabagismo, HT, HPL, DM, acidente vascular cerebral e outros, já foram determinados. Em seus estudos, Deppe et al. e Kim et al. mostraram que fatores cirúrgicos, como técnicas de aquisição de vasos ou uso de circulação extracorpórea, foram preditores independentes de estenose de EVS. ${ }^{18,19}$

O tabagismo é um fator de risco bem conhecido para aterosclerose e doença arterial coronariana. ${ }^{20-22}$ Sen et al. demonstraram que o tabagismo é um fator de risco independente para a doença de EVS em sua análise de regressão multivariada. ${ }^{20-22}$ Semelhante a estudos anteriores, demonstramos que o tabagismo é um importante fator de risco para estenose de EVS.

Demonstrou-se que a DM é uma das principais causas de aterosclerose em diversos estudos. ${ }^{23}$ A DM pode causar estenose de EVS como resultado de disfunção endotelial vascular causada por mecanismos fisiopatológicos imunes e não imunes. ${ }^{24}$ Koshizaka et al. ${ }^{25}$ examinaram a relação entre DM e falha de enxerto avaliada por angiografia de um ano e desfecho clínico de cinco anos em pacientes submetidos a CRM. No estudo, observou-se que que a falha no EVS no primeiro ano foi semelhante entre pacientes com e sem DM. No entanto, óbito, infarto do miocárdio ou revascularização mostraram-se significativamente maiores em pacientes com DM com desfecho clínico de 5 anos. ${ }^{25} \mathrm{Em}$ nosso estudo, histórico de DM foi considerado fator de risco independente para estenose de EVS.

A dislipidemia é um fator de risco bem conhecido para aterosclerose. ${ }^{23}$ Há evidências crescentes de que colesterol HDL baixo e colesterol LDL alto, colesterol total e triglicerídeos desempenham um papel na progressão da aterosclerose e doença arterial coronariana. ${ }^{26}$ Da mesma forma, demonstrou-se que colesterol HDL baixo com colesterol LDL alto está associado à estenose de EVS. ${ }^{27,28}$ Entre esses parâmetros, recomenda-se que o nível de colesterol LDL seja selecionado como alvo do tratamento. ${ }^{26}$ No entanto, após reduzir o colesterol LDL para os níveis recomendados, permanece um risco cardiovascular de $\sim 50 \%$, motivando os pesquisadores a encontrarem novos preditores de arteriosclerose. ${ }^{29}$ Em comparação com os parâmetros lipídicos únicos, os índices lipídicos abrangentes, como IAP, CA e não HDL, são considerados melhores preditores de aterosclerose e doenças arteriais coronarianas. ${ }^{30,31}$

Em nosso estudo, semelhante a estudos anteriores, baixos níveis de colesterol HDL e altos níveis de colesterol LDL foram considerados fatores de risco independentes para estenose de EVS. Além disso, o alto nível de colesterol não HDL, outro fator de risco aterogênico, foi considerado um preditor independente de estenose de EVS.O colesterol não HDL não é o primeiro alvo na terapia hipolipemiante e pode ser um alvo secundário quando os pacientes atingem os níveis recomendados de colesterol LDL. ${ }^{26}$ Em nosso estudo, o nível médio de colesterol LDL dos pacientes foi superior aos valores recomendados para prevenção secundária. Isso mostra que os pacientes não receberam o tratamento hipolipemiante necessário e aqueles que o receberam não usaram doses efetivas para o colesterol LDL, que é o alvo principal.

Como se sabe, a LDL pequena e densa (sdLDL) pode se acumular e oxidar mais facilmente na parede do vaso em comparação com o colesterol LDL. Estudos anteriores mostraram que a sdLDL é um marcador que prediz a aterosclerose e pode ser usado clinicamente. ${ }^{26}$ No entanto, é complicado medir a sdLDL no sangue e seu alto custo limita seu 


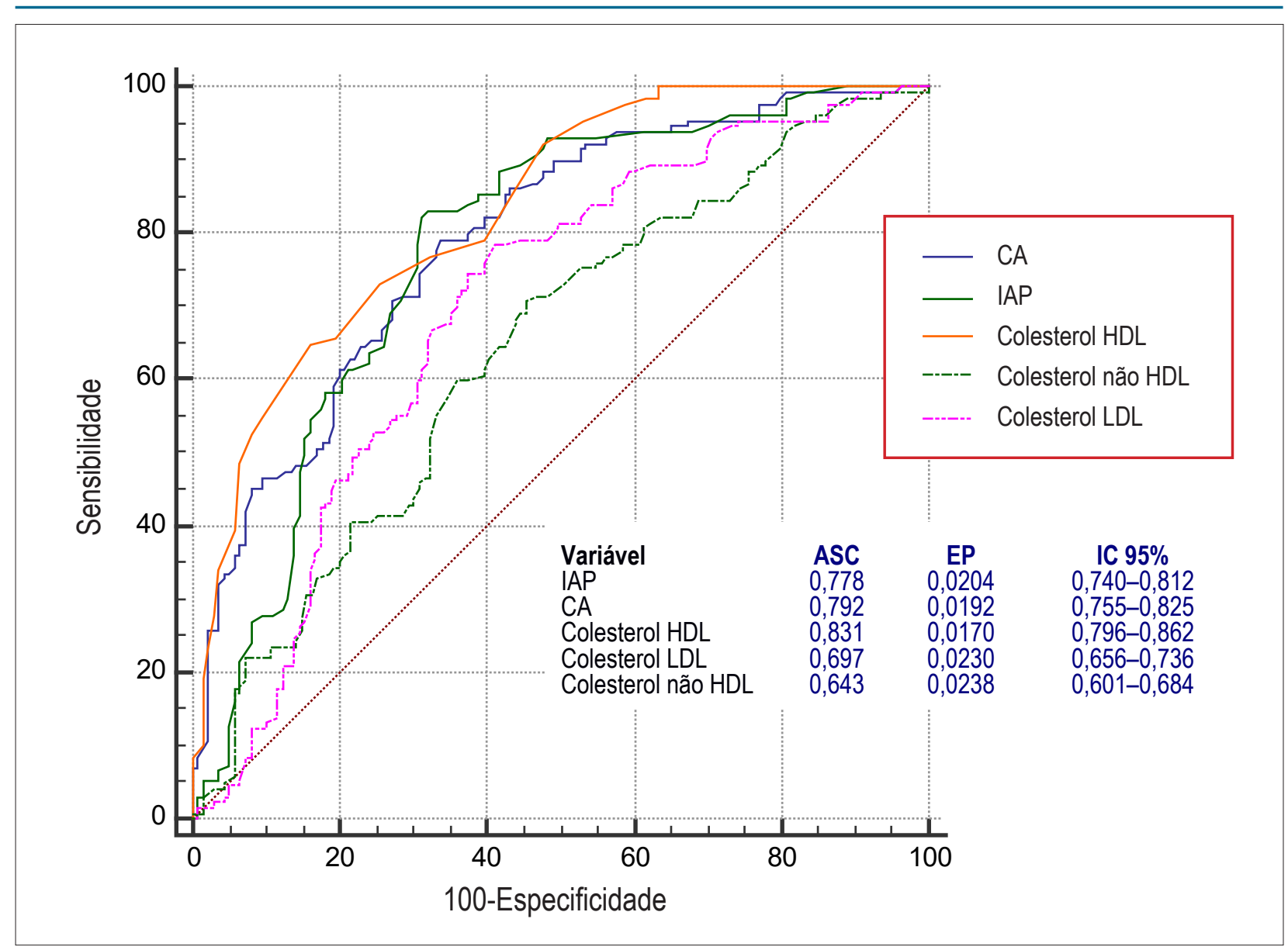

Figura 1 - Curvas receiver operating characteristic (ROC) para estenose de EVS.

uso clínico. O IAP obtido a partir do logaritmo TG/colesterol HDL reflete fortemente o equilíbrio entre lipoproteínas aterogênicas e protetoras. Um estudo anterior mostrou o IAP como inversamente relacionado ao diâmetro do colesterol LDL. ${ }^{32}$ Por esse motivo, o IAP pode ser usado como um fácil indicador na doença arterial coronariana. O IAP também pode ser rotineiramente calculado a partir de parâmetros lipídicos e usado como substituto do tamanho da partícula LDL sem ocasionar custos extras.

No presente estudo, observamos que o IAP é um indicador independente de estenose de EVS e apresentou melhor desempenho na predição de estenose de EVSA do que o colesterol LDL e o colesterol não HLD. Com base nesses resultados, mostramos que o IAP obtido por um cálculo simples pode ser usado para prever estenose de EVS. Em estudos anteriores, os valores médios do IAP diferiram. ${ }^{15,33-35}$ Essas diferenças podem dever-se à não homogeneidade de grupos de pacientes selecionados ou ao uso de fármacos. Outro motivo pode ser a diferença na carga de aterosclerose na população. No presente estudo, avaliamos apenas pacientes submetidos a CRM sob alto risco aterosclerótico. Como resultado, o valor médio do IAP do nosso estudo foi superior ao dos estudos publicados anteriormente.
O coeficiente aterogênico (CA) é uma medida de colesterol que utiliza frações de lipoproteínas LDL-C, VLDL-C e IDL-C em relação ao colesterol HDL. ${ }^{36}$

Ele reflete o potencial aterogênico de todo o espectro de frações de lipoproteínas. Como destacado acima, o colesterol não HDL é o segundo alvo da terapia após o colesterol LDL, de acordo com as diretrizes da ESC (European Society of Cardiology), principalmente em indivíduos com hipertrigliceridemia. ${ }^{26}$ Portanto, esse índice simples pode fornecer informações importantes para a identificação de indivíduos com risco de doença cardiovascular. Em nosso estudo, observamos que o CA médio mostrou-se significativamente maior no grupo estenose EVS (+) do que no grupo EVS (-) e foi preditor independente de estenose.

Além disso, o CA mostrou melhor desempenho na análise ROC do que o colesterol não HDL. Esse resultado mostra que o CA, que é um índice simples, deve ser considerado para prever estenose de EVS.

\section{Limitações}

O presente estudo apresenta algumas limitações. Primeiramente, trata-se de um estudo unicêntrico retrospectivo. No entanto, o número de pacientes é alto, o que aumenta 
o valor do estudo. Em segundo lugar, não comparamos os resultados com indivíduos controle saudáveis, o que poderia mostrar o valor normal do IAP e CA em nossa população saudável. Em terceiro lugar, como não houve desfechos clínicos no estudo, não é possível avaliar o impacto do IAP e do CA nos desfechos clínicos. Portanto, outros estudos são necessários para avaliar o impacto desses índices em pacientes submetidos a CRM.

\section{Conclusão}

No presente estudo, mostramos que HT, DM, acidente vascular cerebral, insuficiência cardíaca e colesterol HDL, colesterol LDL e colesterol não HDL foram preditores independentes de estenose de EVS, semelhante a estudos anteriores. Além disso, até onde sabemos, este é o primeiro estudo que mostra uma associação entre estenose de EVS e os índices aterogênicos IAP e CA. Além disso, observamos que os índices aterogênicos IAP e CA apresentam melhor desempenho na predição de estenose de EVS do que os parâmetros lipídicos padrão colesterol LDL, colesterol HDL e colesterol não HDL.

\section{Referências}

1. Lee MS, Park SJ, Kandzari DE, Kirtane AJ, Fearon WF, Brilakis ES, et al. Saphenous vein graft intervention. JACC Cardiovasc Interv. $2011 ; 4(8): 831-43$.

2. Goldman S, Zadina K, Moritz T, Ovitt T, Seth G, Copeland JG, et al. Longterm patency of saphenous vein and left internal mammary artery grafts after coronary artery bypass surgery: results from a department of veterans affairs cooperative study. J Am Coll Cardiol. 2004;44(11):2149-56.

3. Allaire E, Clowes AW. Endothelial cell injury in cardiovascular surgery: the intimal hyperplastic response. Ann Thorac Surg. 1997;63(2):582-91.

4. Wiedemann D, Kocher A, Bonaros N, Semsroth S, Laufer G, Grimm M, et al. Perivascular administration of drugs and genes as a means of reducing vein graft failure. Curr Opin Pharmacol. 2012;12(2):203-16.

5. Motwani JG, Topol EJ. Aortocoronary saphenous vein graft disease: pathogenesis, predisposition, and prevention. Circulation. 1998;97(9):916-31.

6. Boyle EM Jr, Lille ST, Allaire E, Clowes AW, Verrier ED. Endothelial cell injury in cardiovascular surgery: atherosclerosis. Ann Thorac Surg. 1997;63(3):885-94

7. Kannel WB, Neaton JD, Wentworth D, Thomas HE, Stamler J, Hulley SB, et al. Overall and coronary heart disease mortality rates in relation to major risk factors in 325,348 men screened for the MRFIT. Multiple risk factor intervention trial. Am Heart J. 1986;112(4):825-36.

8. Castelli WP, Garrison RJ, Wilson PW, Abbott RD, Kalousdian S, Kannel WB. Incidence of coronary heart disease and lipoprotein cholesterol levels: the Framingham Study. JAMA. 1986;256(20):2835-8.

9. Miller GJ, Miller NE. Plasma-high-density-lipoprotein concentration and development of ischaemic heart disease. Lancet. 1975;1(7897):16-9.

10. Castelli WP. The triglyceride issue: a view from Framingham. Am Heart J. $1986 ; 112(2): 432-7$

11. Ridker PM, Rifai N, Cook NR, Bradwin G, Buring JE. Non-HDL cholesterol, apolipoprotein A-I and B100, standard lipid measures, lipid ratios, and CRP as risk factors for cardiovascular disease in women. JAMA. 2005;294(3):326-33.

12. Kinosian B, Glick H, Garland G. Cholesterol and coronary heart disease: predicting risks by levels and ratios. Ann Intern Med. 1994;121(9): 641-7.

\section{Contribuição dos autores}

Concepção e desenho da pesquisa: Yavuz F; Obtenção de dados: Kaplan M, Yıldırım A; Análise e interpretação dos dados: Kaplan M, Yıldırım A, Dogdus M; Análise estatística: Kilic S; Obtenção de financiamento: Kucukosmanoglu M; Redação do manuscrito: Yavuz F; Revisão crítica do manuscrito quanto ao conteúdo intelectual importante: Yavuz F, Kilic S.

\section{Potencial conflito de interesses}

Declaro não haver conflito de interesses pertinentes.

\section{Fontes de financiamento}

O presente estudo não teve fontes de financiamento externas.

\section{Vinculação acadêmica}

Não há vinculação deste estudo a programas de pósgraduação.

13. Cui Y, Blumenthal RS, Flaws JA, Whiteman MK, Langenberg P, Bachorik PS, et al. Non-high-density lipoprotein cholesterol level as a predictor of cardiovascular disease mortality. Arch Intern Med. 2001;161(11):1413-9.

14. Wu J, Chen S, Liu L, Gao X, Zhou Y, Wang C, et al. Non-high-density lipoprotein cholesterol vs low-density lipoprotein cholesterol as a risk factor for ischemic stroke: a result from the Kailuan study. Neurol Res. 2013;35(5):505-11

15. Dobiasova M. Atherogenic index of plasma [ $\log$ (triglycerides/HDLcholesterol)]: theoretical and practical implications. Clin Chem. 2004;50(7):1113-5

16. Dobiasova M, Frohlich J. The plasma parameter log (TG/HDL-C) as an atherogenic index: correlation with lipoprotein particle size and esterification rate inapob-lipoprotein-depleted plasma (FERHDL). Clin Biochem. 2001;34(7):583-8.

17. Deric M, Kojic-Damjanov S, Cabarkapa V, Eremic N. Biochemical markers of atherosclerosis. JMB. 2008;27(2):148-53

18. Deppe AC, Arbash W, Kuhn EW, Slottosch I, Scherner M, Liakopoulos OL, et al. Current evidence of coronary artery bypass grafting off-pump versus on-pump: a systematic review with meta-analysis of over 16,900 patients investigated in randomized controlled trials. Eur J Cardiothorac Surg. 2016;49(4):1031-41.

19. Kim YH, Oh HC, Choi JW, Hwang HY, Kim KB. No-touch saphenous vein harvesting may improve further the patency of saphenous vein composite grafts: early outcomes and 1-year angiographic results. Ann Thorac Surg. 2017;103(5):1489-97.

20. Muir AD, McKeown PP, Bayraktutan U. Role of gender, smoking profile, hypertension, and diabetes on saphenous vein and internal mammary artery endothelial relaxation in patients with coronary artery bypass grafting. Oxid Med Cell Longev. 2010;3(3):199-205.

21. Higman DJ, Greenhalgh RM, Powell JT. Smoking impairs endotheliumdependent relaxation of saphenous vein. Br J Surg. 1993;80(10):1242-5.

22. Sen F, Yilmaz S, Balci KG, Sen O, Gül M, Çay S, et al. The relationship between vitamin $\mathrm{D}$ levels and saphenous vein graft patency. Coron Artery Dis. 2015;26(4):328-32 
23. Piepoli MF, Hoes AW, Agewall S, Albus C, Brotons C, Catapano AL, et al. 2016 European Guidelines on Cardiovascular Disease Prevention in Clinical Practice: The Sixth Joint Task Force of the European Society of Cardiology and Other Societies on Cardiovascular Disease Prevention in Clinical Practice (Constituted by Representatives of 10 Societies and by Invited experts)Developed With the Special Contribution of the European Association for Cardiovascular Prevention \& Rehabilitation (EACPR). Eur Heart J. 2016;37(29):2315-81.

24. Radenkovic M. Pioglitazone and endothelial dysfunction pleiotropic effects and possible therapeutic implications. Sci Pharm. 2014;82(4):709-21.

25. Koshizaka M, Lopes RD, Reyes EM, Gibson CM, Schulte P, Hafley GE, et al. Long-term clinical and angiographic outcomes in patients with diabetes undergoing coronary artery bypass graft surgery: results from the project of Ex-vivo Vein graft engineering via transfection IV trial. Am Heart J. 2015;169(1):175-84.

26. Catapana AL, Graham I, Backer G, Wiklund O, Chapman MJ, Drexel H, et al. 2016 ESC/EAS Guidelines for the Management of Dyslipidaemias: The Task Force for the Management of Dyslipidaemias of the European Society of Cardiology (ESC) and European Atherosclerosis Society (EAS) developed with the special contribution of the European Assocciation for Cardiovascular Prevention \& Rehabilitation (EACPR). Atherosclerosis. 2016 Oct; 253:281344.

27. Jerzewski K, Ruel M, Voisine P, May MR, Kulik A. Does high-density lipoprotein influence the development of saphenous vein graft disease after coronary bypass surgery?: exploratory analysis from the CASCADE trial. J Cardiothorac Surg. 2013 Jul 10;8:172.

28. Hata M, Takayama T, Sezai A, Yoshitake I, Hirayama A, Minami K. Efficacy of aggressive lipid controlling therapy for preventing saphenous vein graft disease. Ann Thorac Surg. 2009;88(5):1440-4.
29. Ridker PM, Rifai N, Rose RL, Buring JE, Cook NR. Comparison of C-reactive protein and low-density lipoprotein cholesterol levels in the prediction of first cardiovascular events. N Engl J Med. 2002;347(20):1557-65.

30. Gao M, Zheng Y, Zhang W, Cheng Y, Wang L, Qin L. Non-high-density lipoprotein cholesterol predicts nonfatal recurrent myocardial infarction in patients with ST segment elevation myocardial infarction. Lipids Health Dis. 2017 Jan 23;16:20.

31. Zhu L, Lu Z, Zhu L, Ouyang X, Yang Y, He W, et al. Lipoprotein ratios are better than conventional lipid parameters in predicting coronary heart disease in Chinese Han people. Kardiol Pol. 2015;73(10):931-8.

32. Frohlich J, Dobiasova M. Fractional esterification rate of cholesterol and triglycerides to $\mathrm{HDL}$-cholesterol are powerful predictors of positive findings on coronary angiography. Clin Chem. 2003;49(11):1873-80.

33. Nansseu JRN, Moor VJA, Nouaga MED, Zing-Awona-B, Tchanana G, Ketcha A. Atherogenic index of plasma and risk of cardiovascular disease among Cameroonian postmenopausal women. Lipids Health Dis. 2016 Mar 9;15:49.

34. Hartopo AB, Arso IA, Setianto BY. Low plasma atherogenic index associated with poor prognosis in hospitalized patients with acute myocardial infarction. Acta Med Indones. 2016;48(2):106-13.

35. Wang YM, Chen XF, Yuan Q. Analysis on levels of non-high density lipoproterin cholesterol and atherogenic index of plasma in normal population. Zhejiang Prev Med. 2008;1:10-4.

36. Hermans MP, Sacks FM, Ahn SA, Rousseau MF. Non-HDL-cholesterol as valid surrogate to apolipoprotein B100 measurement in diabetes: discriminant ratio and unbiased equivalence. Cardiovasc Diabetol. 2011 Feb 28;10:20. 